\title{
Managing a mixed-registration-type appointment system in outpatient clinics
}

\author{
Syi Su*, Chung-Liang Shih
}

\author{
Institute of Health Care Organization Administration, School of Public Health, National Taiwan University, \\ No. 1, Sec. 1, Jen Ai Rd, Rm. 1512, Taipei 100, Taiwan, ROC \\ Department of Emergency Medicine, National Taiwan University Hospital, Taipei 100, Taiwan, ROC
}

Received 17 July 2002; received in revised form 30 October 2002; accepted 19 December 2002

\author{
KEYWORDS \\ Mixed-registration-type; \\ Healthcare organizations; \\ Alternative models; \\ Simulation
}

\begin{abstract}
Summary Introduction: Improving outpatient resource utilization significantly enhances the efficiency of healthcare organizations. Substantial number of walk-in patients (average of $72 \%$ in our study) to outpatient services is a universal characteristic of Taiwan's healthcare organizations. Consequently, scheduling becomes extremely complicated and important. Selecting the right scheduling alternative, a healthcare organization can markedly improve operating efficiency of outpatient resources. Objective: This research applied simulation methodology to analyze several scheduling solutions and found that setting the appropriate arrival time interval for preregistered patients significantly impacts queuing problems in outpatient services. Method: Using established simulation models, the effects of various scheduling policies on patients' throughput time and waiting times were revealed. Under alternative model A, the first 20 numbers are reserved for scheduled patients; after that, only even numbers are offered for scheduled ones. Odd numbers after $\mathbf{2 0}$ are left for walk-ins. Under alternative model $\mathrm{B}$, front numbers were assigned to scheduled patients successively. The later numbers were left for walk-ins. Alternative model $C$ assigned scheduled patients with even numbers and walk-ins with odd numbers in sequence. Finally, alternative model $D$ was designed to examine the optimal scheduled time interval by conducting the model with different scheduled time intervals such as 3, 5, 7, 9, and 11 min. Result: The alternative sequence (alternative model $\mathrm{C}$ - assigning even numbers for scheduled patients and odd numbers for walk-in patients, or vice versa) significantly has the least throughput time (average: $34.9 \mathrm{~min}$ vs 55.2, 56.2, and $46.2 \mathrm{~min}$ ) and waiting times (average: 14.7 min vs $34.9,35.8$, and $25.8 \mathrm{~min}$ ) for walk-in patients compared with other registration strategies. Scheduling the appointments with flexible time interval (alternative model D) has the least throughput time (average: $24.2 \mathrm{~min}$ vs $28.4,28.2$, and 37.2 $\mathrm{min}$ ) and waiting times (average: $8.0 \mathrm{~min}$ vs 12.5, 12.3, and $20.5 \mathrm{~min}$ ) for scheduled patients compared with other registration strategies. Conclusion: The findings of this research could be applied possibly to any outpatient clinic with mixed-registrationtype (walk-in and scheduled), particularly which accounts for high percentage of walk-in patients.
\end{abstract}

(C) 2003 Elsevier Science Ireland Ltd. All rights reserved.

\footnotetext{
*Corresponding author. Present address: Institute of Health Care Organization Administration, School of Public Health, National Taiwan University, No. 1, Sec. 1, Jen Ai Rd, Rm. 1512, Taipei 100, Taiwan, ROC. Tel./fax: +886-2-2341-2520.

E-mail address: susyi@ha.mc.ntu.edu.tw (S. Su).
} 


\section{Introduction}

Competition among healthcare organizations has become intense, especially for outpatient services in Taiwan. Increasingly, traditional inpatient services are provided on an outpatient basis because of technology advancement as well as the demands of national health insurance (NHI) [1]. As a result, outpatient services play a major role in healthcare delivery. Two-thirds of Taiwan's total NHI expenditure is currently spent on outpatient services. Hence, managing outpatient services is of great importance to healthcare organizations.

Since people are free to select their healthcare providers under $\mathrm{NHI}$, walk-in patients are common in most outpatient clinics in Taiwan. Therefore, managers of these outpatient clinics may face the following problems: (1) they need to maintain high levels of personnel and equipment to provide services within a limited period of time; (2) they must employ diverse professionals such as physicians, nurses, social workers, administrators, etc. (however, patients are prone to select other clinics if, during visits, they encounter too lengthy waiting or throughput times); and (3) managers must predict exactly how many patients will come to the outpatient clinics and at what time. It is nearly impossible to predict these if outpatient clinics lack an optimal scheduling system. Thus, it becomes most important and quite complicated to manage a scheduling system because most patients prefer to walk-in directly instead of getting an appointment. For example, the walk-in rate is $72 \%$ compared with a $28 \%$ scheduled rate of subject clinics in this research study.

Operations research and management techniques have been applied to healthcare organizations to gain insight into the results of restructuring or reengineering operation systems. Previous studies have used motion-time method (MTM) studies, queuing models [2-6], and simulation models to improve the healthcare delivery process. MTM studies are usually applied to explore each specific activity movement, while queuing studies are aimed to target system's waiting behavior or waiting line, but is restricted by some theoretical assumptions. However, both MTM and queuing models ignore interactions among subsystems, although they can clearly define the healthcare delivery process. Computer simulation allows much more accurate modeling of these systems, including transient conditions based on random patient arrival and service time with realistic statistical distribution, and can more potentially reveal the results of various alternatives, although it also requires more theoretical and technical learning.
Some authors employ simulation methods to model the complex and general systems successfully [720]. They not only provide alternative solutions to a particular problem in the systems, but also evaluate human resources, equipment utilization, process change, and benefits in the rapidly expanding health delivery field. Thus, simulation is a useful tool to analyze and exhibit the results of system alternations, instead of utilizing trial and error processes to reach optimal policy, saving both administrator and patient exertion.

This study proposes a better scheduling philosophy by showing how a simulation model can be applied to outpatient clinics that provide registration for both the scheduled and walk-in patients, and recommends the best feasible solution to this kind of system. Several simulated alternatives demonstrate the impact on outcomes such as patient waiting time and throughput time.

\section{Materials and methods}

All clinics located in Su-Ten Urology Hospital are specialty for urology services. They have totally 11 consultation sections per week from 8:30 A.M. to 5:30 P.M. on weekdays and from 8:30 A.M. to 12:30 P.M. on Saturdays. In every 4-h consultation period, various numbers of clinics are open for patient consultation based on the availability of physicians and amount of historical patient flow, such as three clinics open in the morning of Monday, Wednesday, and Friday, four clinics open in the morning of Tuesday, Thursday, and Saturday, etc. The flowchart of outpatient visit is illustrated in Fig. 1.

The subject hospital is a private for-profit urological specialty hospital and provides about $56,000-60,000$ ambulatory visits annually. The urology clinics are staffed with five full-timeequivalent (FTE) physicians, four administrators, and three nurses. The patient load averages 20 in each clinic per consultation section (morning or afternoon). An individual physician appointment system allows patients to self-select specific physicians for consultation. The clinic also allows for walk-in registration. The current patient scheduling policy is as follows: the first 20 numbers are reserved for scheduled patients; after that, only even numbers are offered for scheduled ones. Odd numbers after 20 are left for walk-ins. The appointed arrival time is assigned to those scheduled ones. The arrival time of the first patient is assumed to be the same as the clinic starting time. The scheduled patients are assigned based on 3min intervals and are also informed about their appointed arrival time. If the scheduled patient 


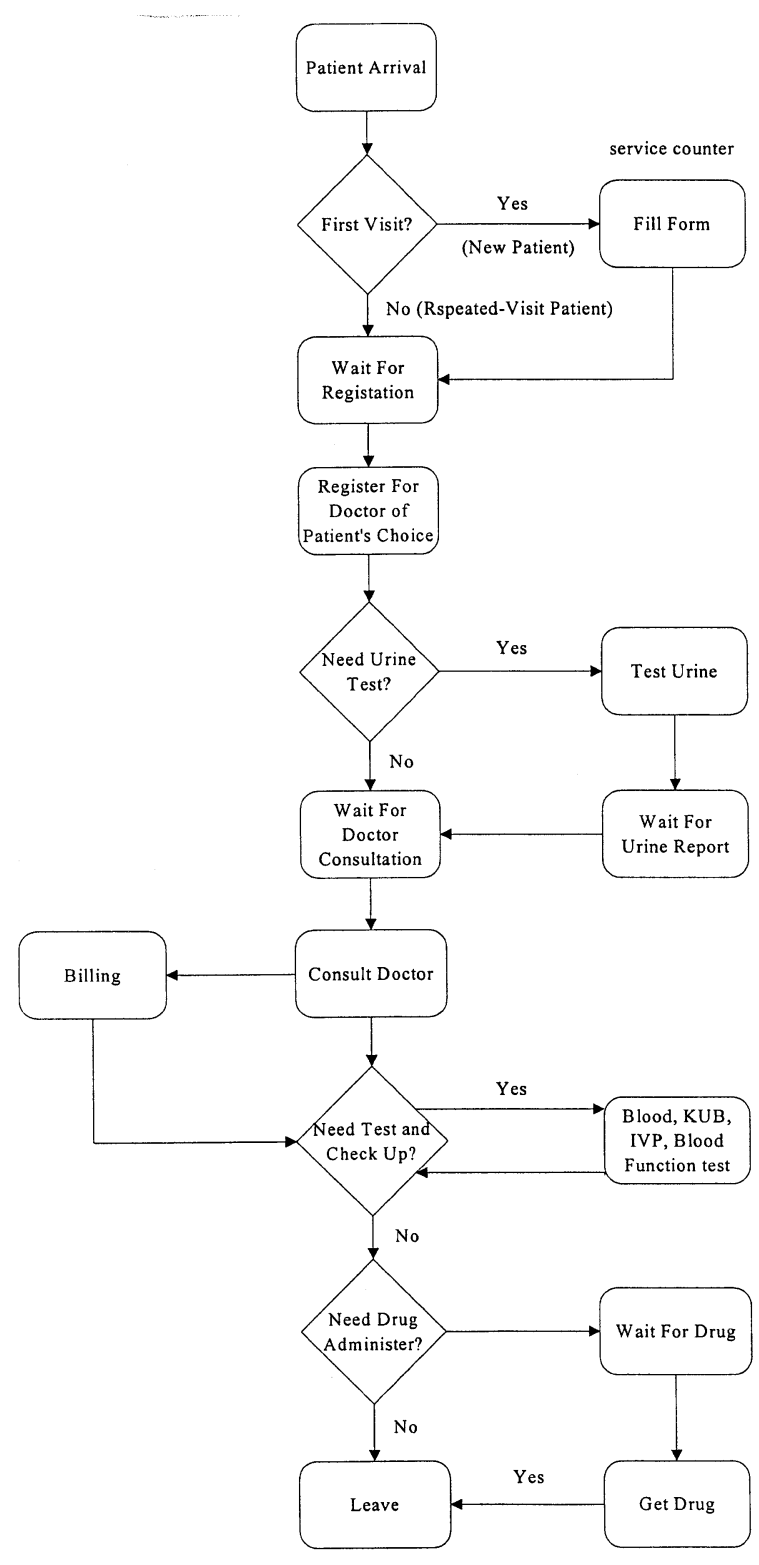

Fig. 1 Flow chart of outpatient visit in subject clinic.

does not appear on time for his/her turn, the next available patient receives consultation immediately. This operating philosophy of services is the so-called "first in, first seen" in order to limit patient waiting time. Under this system, patients believe that they can be seen earlier if they come earlier. Therefore, the patient would rather walkin to see a physician than showed up at the appointed time. The benefits of a scheduling system cannot be achieved since patients have no incentive to keep their appointed time.

The discrete process-based simulation model was created using the MedModel software package as a discrete event simulator [21], which is a Windows-based simulation tool for simulating and analyzing healthcare systems of all types and sizes.
The process-based simulation is to take the whole process of an entity as the basic logical building block of a simulation model. The life of an entity is traced by checking its progress through its process. Models are constructed through a series of ease-ofuse modules and predefined operation logics such as through patient movement, flow, and care plan logic. The convenient modeling constructs and graphical user interface such as locations, resources, processing, arrival, entities, etc. are used to build a particular system and test new ideas for system design or improvement before committing the time and resources necessary to build or alternate the actual system. "Locations" represent fixed spaces in the system where entities are routed for processing. "Resources" may be persons, pieces of equipment, or some other devices used for any one or more entities or locations. "Processing" defines everything that happens to an entity from the time it enters a system. Any time new entities are introduced into the system, it is called an "arrival". Anything that a model processes is called an "entity" such as patients, documents, etc. Full programming capability is provided for applications requiring complex logic [21]. An animation of the operation process (processing), patients (entity), personnel pathway, and of resource changeover time was presented on a computer monitor. After the simulation, performance measures such as resource utilization, system capacity, and capability are tabulated for evaluation.

The input data for model building - probability distributions of interarrival times and service times for each section-were fitted from empirical observation of time-motion study conducted from January 6 to February 4, 2000. Other input information was abstracted from the patients' registration file including patient load, number of kept and broken appointments, and on-site registration numbers. Besides, 544 cases were sampled randomly and observed from a total of 4673 visits during study period to produce the input data needed for model building, such as service times among different activities. The model was built on a "walk-through process" and time frame. The service logic in this model is patient-centered. Two downtimes are incorporated for the clinics and physicians, but physician downtimes have a lower priority than those of clinics. Thus, patients can register before a clinic's downtime, but physicians can only begin downtime after all registered patients are served.

The probability distribution of variant serving times necessary to built this model, such as the testing time and the physician consulting time, was 
determined by BestFit [22], a tool used for data fitting. The goodness-of-fit statistic was made in Kolmogorov-Simirnov test. The desired output included physician consultation time, physical test processing time, and administering service time. Output performance indicators included the patient's waiting time, throughput time, and the utilization rate for various personnel and resources. Eight specialists familiar with this system worked in the clinics and were asked to view the animation and verify the model. Model validation was done finally by making statistical comparisons between simulation outputs (e.g. throughput time, waiting time) and the empirical data under the present assumptions (Tables 1 and 2). Throughput time includes registration, waiting time, treatment, and prescriptions.

The model was used to experiment with several scheduling policies. Under alternative model A, scheduled patients were supposed to arrive on the appointed time, and physicians consulted them according to the registered numbers instead of "first in, first seen". Under alternative model B, front numbers were assigned to scheduled patients successively. The later numbers were left for walkins. Alternative model $\mathrm{C}$ assigned scheduled patients with even numbers and walk-ins with odd numbers in sequence. Finally, alternative model $D$ was designed to examine the optimal scheduled time interval by conducting the current model with different scheduled time intervals such as 3, 5, 7, 9 , and $11 \mathrm{~min}$. The suitable time interval was defined as having two criteria: (1) the total throughput time is less than that of alternative model A and (2) the ratio of actual working hours to the clinics' office hours (utilization rate) should be between 70 and $90 \%$. In order to obtain the optimal estimate, 100 simulation runs were replicated for each alternative. These output results were analyzed and compared with those from the current policy. Student's $t$-test was utilized for statistical comparisons at a significance level of $0.05 \alpha$-value. Because there were five proposed alternative models (current and alternative models A, B, C, and D), we made 10 comparisons. Consequently, we divided the original $\alpha=0.05$ significance level by 10 to incorporate multiple statistical allowances. Therefore, we set the significance level required by each pair of comparisons at $0.005(0.05 / 10)$.

\section{Results}

A snapshot observation of the visual interactive modeling system built with MedModel software is shown in Fig. 2. The theoretical probability distribution of various patient arrivals and service times was input. Based on the result of goodnessof-fit test, Poisson, beta, and negative binomial distributions were chosen for majority of patient arrival patterns in all sections. Gamma, log-normal, Pearson, and Weibull distributions were accounted for majority of processing times through the simulated model.

The throughput time and waiting time of patients in each visit section are included in Tables 3 and 4. Means of throughput and waiting times in the 11 visit sections for scheduled patients are $35.1 \pm 6.2$ and $18.8 \pm 6.2 \mathrm{~min}$, respectively. For walk-in patients, the mean times are $39.4 \pm 5.7$ and $19.5 \pm 5.4 \mathrm{~min}$.

In alternative model $A$, researchers attempted to regulate patient's visit order in accordance with the assigned numbers instead of the "first in, first seen" rule. The mean throughput and waiting times are $28.4 \pm 3.4$ and $12.5 \pm 3.0$ min for scheduled patients and are $55.2 \pm 12.0$ and $34.9 \pm 11.3$ min for walk-ins (see Tables 3 and 4). The results revealed that scheduled patients took less time than patients who registered walk-in regardless of the throughput time or waiting time. For scheduled patients, the throughput and waiting times of alternative model A were significantly less than the current ones $(P<0.01$; see Tables 5 and 6$)$, but both throughput time and waiting time for walk-ins were significantly longer than the current situation $(P<0.01$; see Tables 5 and 6$)$.

Table 1 Validation of waiting time for physician between simulated results and empirical data

\begin{tabular}{|c|c|c|c|c|c|}
\hline & \multicolumn{2}{|c|}{ Empirical data } & \multicolumn{2}{|c|}{ Simulation results } & \multirow[t]{2}{*}{$P$-value } \\
\hline & Mean (min) & S.D. & Mean (min) & S.D. & \\
\hline New patients & 35.5 & 16.9 & 39.9 & 22.4 & 0.3 \\
\hline Walk-in patients & 23.6 & 16.2 & 22.0 & 16.2 & 0.5 \\
\hline Walk-in patients receiving routine urinalysis & 38.2 & 14.9 & 33.6 & 17.6 & 0.2 \\
\hline Scheduled patients & 23.3 & 27.5 & 19.7 & 17.1 & 0.2 \\
\hline Scheduled patients receiving routine urinalysis & 36.2 & 15.2 & 37.4 & 21.9 & 0.6 \\
\hline
\end{tabular}


Table 2 Throughput time validation between simulated results and empirical data

\begin{tabular}{|c|c|c|c|c|c|}
\hline & \multicolumn{2}{|c|}{ Empirical data } & \multicolumn{2}{|c|}{ Simulation results } & \multirow[t]{2}{*}{$P$-value } \\
\hline & Mean (min) & S.D. & Mean (min) & S.D. & \\
\hline Patients requiring no test & 43.4 & 26.1 & 40.9 & 27.4 & 0.3 \\
\hline Patients receiving blood test & 48.2 & 15.7 & 45.4 & 21.6 & 0.7 \\
\hline Patients receiving $\mathrm{X}$-ray check up & 61.9 & 20.3 & 53.3 & 18.6 & 0.1 \\
\hline Patients receiving bladder function test & 75.6 & 27.1 & 63.8 & 24.5 & 0.2 \\
\hline
\end{tabular}

In alternative models $B$ and $C$, researchers rearranged the sequence of registered numbers assigned to scheduled and walk-in patients. The alternative model $B$ assigned scheduled patients with previous numbers. The later numbers were assigned to the walk-ins in sequence. Simulation outputs revealed no significant difference for scheduled patients on both throughput time and waiting time between alternative models $A$ and $B$ $(P=0.87$ and 0.85 , respectively; see Tables 5 and 6). Comparing alternative model $B$ with the current situation for both throughput time and waiting time, alternative model B was shorter than the current situation for scheduled patients but longer than current situation for walk-ins. The same result is found between alternative model $A$ and the current situation (see Tables 5 and 6). Both throughput and waiting times of alternative models $A$ and $B$ for the scheduled patients were still less than those of the walk-ins (see Tables 3 and 4). Another appointment policy, alternative model C, assigned scheduled patients with even numbers and walk-ins with odd numbers in sequence. The throughput time and the waiting time in alternative model $\mathrm{C}$ were not significantly different from the current situation for walk-in patients $(P=0.057$ and 0.042 , respectively), and there was no significant difference for scheduled patients $(P=0.46$ and 0.50 , respectively; see Tables 5 and 6 ).

The researchers performed a sensitivity analysis on the physician visit interval for scheduled patients. The hypothesis was that doctors could serve walk-in patients better by lengthening scheduled time intervals. Alternative model $D$ explored the most suitable time interval by simulating different appointment interval values such as 3 , $5,7,9$, and $11 \mathrm{~min}$. Based on the results of 100 replicated simulations, the recommended scheduled time interval for the 11 clinic sections is shown in Table 7. In terms of the above-mentioned criteria, the suggested appointment time interval for scheduled patients was simulated. Using the 11

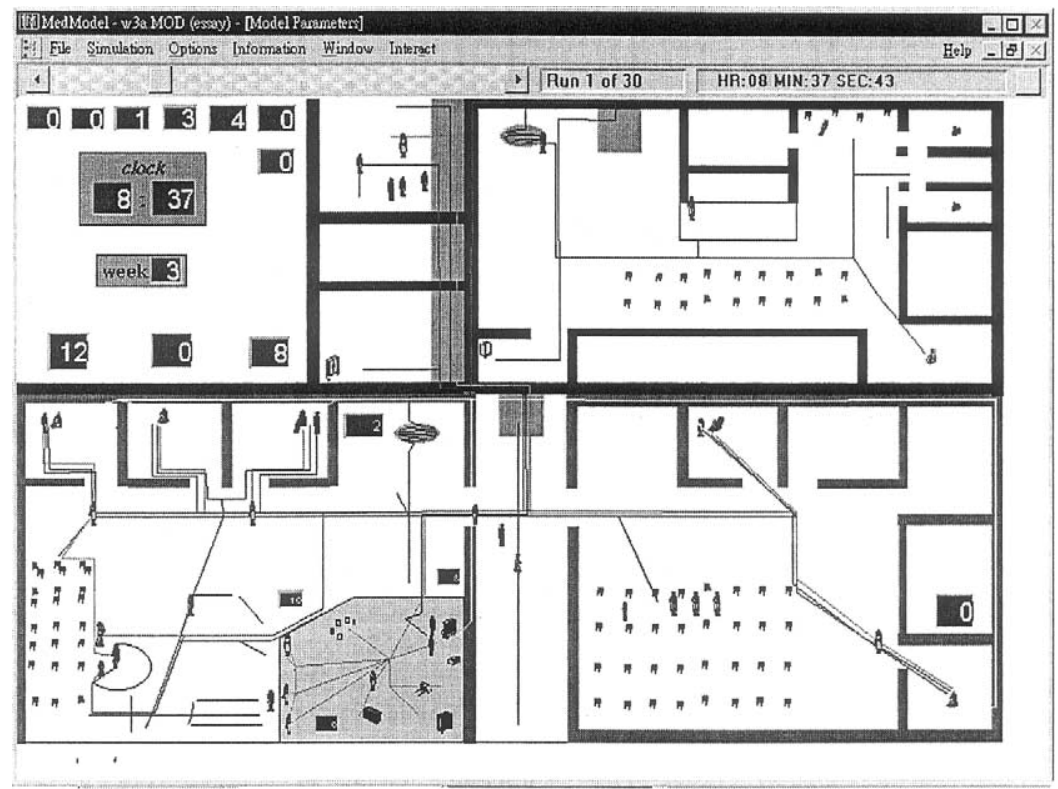

Fig. 2 Windows of studied simulation software and layout of simulation model. Upper right: waiting area and examination room (basement level). Upper left: system time and sequence numbers for consultation and examination. Lower right: waiting area and examination rooms (basement level). Lower left: waiting area for registration and physician consultation (floor level). 
Table 3 Throughput time under current situation and alternative models A-D

\begin{tabular}{|c|c|c|c|c|c|c|c|c|c|c|}
\hline \multirow[t]{2}{*}{ Time } & \multicolumn{2}{|c|}{ Current situation (min) } & \multicolumn{2}{|c|}{ Model A (min) } & \multicolumn{2}{|c|}{ Model B (min) } & \multicolumn{2}{|c|}{ Model C (min) } & \multicolumn{2}{|c|}{ Model D (min) } \\
\hline & Scheduled & Walk-in & Scheduled & Walk-in & Scheduled & Walk-in & Scheduled & Walk-in & Scheduled & Walk-in \\
\hline Monday (A.M.) & 30.1 & 35.9 & 27.8 & 46.0 & 27.6 & 46.5 & 35.2 & 32.8 & 19.9 & 41.7 \\
\hline Monday (P.M.) & 38.4 & 42.2 & 27.1 & 66.7 & 26.5 & 67.2 & 38.7 & 36.8 & 25.1 & 52.0 \\
\hline Tuesday (A.M.) & 36.6 & 39.5 & 27.9 & 56.3 & 27.9 & 56.3 & 40.2 & 31.1 & 25.7 & 46.5 \\
\hline Tuesday (P.M.) & 45.2 & 52.1 & 34.8 & 78.0 & 34.1 & 78.3 & 48.7 & 46.1 & 21.5 & 55.5 \\
\hline Wednesday (A.M.) & 24.6 & 30.1 & 24.2 & 34.1 & 24.2 & 34.1 & 26.6 & 29.6 & 18.8 & 32.0 \\
\hline Wednesday (P.M.) & 34.3 & 38.3 & 26.6 & 61.8 & 27.0 & 61.7 & 36.9 & 35.6 & 26.0 & 47.8 \\
\hline Thursday (A.M.) & 32.7 & 38.3 & 26.4 & 52.2 & 26.2 & 52.1 & 38.2 & 31.2 & 28.6 & 41.6 \\
\hline Thursday (P.M.) & 34.6 & 38.1 & 25.0 & 50.9 & 25.2 & 51.0 & 29.9 & 39.6 & 20.5 & 47.6 \\
\hline Friday (A.M.) & 29.6 & 35.4 & 27.2 & 45.4 & 27.3 & 46.1 & 32.1 & 33.0 & 22.4 & 41.9 \\
\hline Friday (P.M.) & 34.9 & 38.2 & 33.3 & 51.8 & 32.3 & 57.4 & 34.2 & 34.7 & 24.5 & 47.3 \\
\hline Saturday (A.M.) & 44.8 & 44.9 & 32.1 & 64.2 & 31.5 & 67.2 & 48.6 & 33.0 & 32.8 & 54.8 \\
\hline Average & 35.1 & 39.4 & 28.4 & 55.2 & 28.2 & 56.2 & 37.2 & 34.9 & 24.2 & 46.2 \\
\hline
\end{tabular}

Model A: the first 20 numbers reserved for scheduled patients followed by even numbers only; model B: privileged numbers for scheduled ones and last numbers for walk-ins; model C: scheduled/walk-ins in alternative sequence; model D: scheduled patients assigned in successive by various time intervals.

Table 4 Waiting time under current situation and models A-D

\begin{tabular}{|c|c|c|c|c|c|c|c|c|c|c|}
\hline \multirow[t]{2}{*}{ Time } & \multicolumn{2}{|c|}{ Current situation (min) } & \multicolumn{2}{|c|}{ Model A (min) } & \multicolumn{2}{|c|}{ Model B (min) } & \multicolumn{2}{|c|}{ Model C (min) } & \multicolumn{2}{|c|}{ Model D (min) } \\
\hline & Scheduled & Walk-in & Scheduled & Walk-in & Scheduled & Walk-in & Scheduled & Walk-in & Scheduled & Walk-in \\
\hline Monday (A.M.) & 13.9 & 16.7 & 11.8 & 27.4 & 11.767 & 27.6 & 19.0 & 13.7 & 4.5 & 22.3 \\
\hline Monday (Р.м.) & 23.5 & 21.0 & 12.2 & 44.1 & 11.5 & 44.9 & 22.0 & 16.0 & 9.3 & 30.1 \\
\hline Tuesday (A.M.) & 20.0 & 19.1 & 11.5 & 34.5 & 11.4 & 34.5 & 23.0 & 10.2 & 9.1 & 25.5 \\
\hline Tuesday (P.M.) & 29.3 & 32.2 & 19.3 & 58.1 & 18.7 & 58.4 & 31.9 & 26.7 & 6.4 & 35.7 \\
\hline Wednesday (A.M.) & 8.0 & 11.3 & 8.7 & 15.2 & 8.7 & 15.2 & 11.6 & 10.2 & 3.6 & 13.2 \\
\hline Wednesday (P.M.) & 19.2 & 17.9 & 11.3 & 39.2 & 11.8 & 39.9 & 20.0 & 14.6 & 10.3 & 26.4 \\
\hline Thursday (A.M.) & 16.4 & 18.4 & 11.0 & 32.5 & 10.8 & 32.8 & 20.9 & 10.9 & 10.6 & 20.3 \\
\hline Thursday (P.M.) & 19.1 & 18.9 & 10.7 & 31.9 & 10.9 & 32.0 & 15.5 & 20.0 & 6.0 & 28.6 \\
\hline Friday (A.M.) & 12.5 & 15.8 & 10.2 & 25.4 & 10.6 & 26.0 & 15.8 & 13.2 & 5.7 & 21.9 \\
\hline Friday (P.M.) & 18.0 & 17.8 & 16.3 & 32.1 & 15.3 & 35.9 & 17.9 & 14.3 & 8.7 & 26.4 \\
\hline Saturday (A.M.) & 26.4 & 25.0 & 14.3 & 43.5 & 13.5 & 46.4 & 28.0 & 12.2 & 13.6 & 33.6 \\
\hline Average & 18.8 & 19.5 & 12.5 & 34.9 & 12.3 & 35.8 & 20.5 & 14.7 & 8.0 & 25.8 \\
\hline
\end{tabular}

Model A: the first 20 numbers reserved for scheduled patients followed by even numbers only; model B: privileged numbers for scheduled ones and last numbers for walk-ins; model C: scheduled/walk-ins in alternative sequence; model D: scheduled patients assigned in successive by various time intervals. 
Table 5 Comparison matrix of throughput time using Student's $t$-test ( $P$-value) for current situation and models A-D

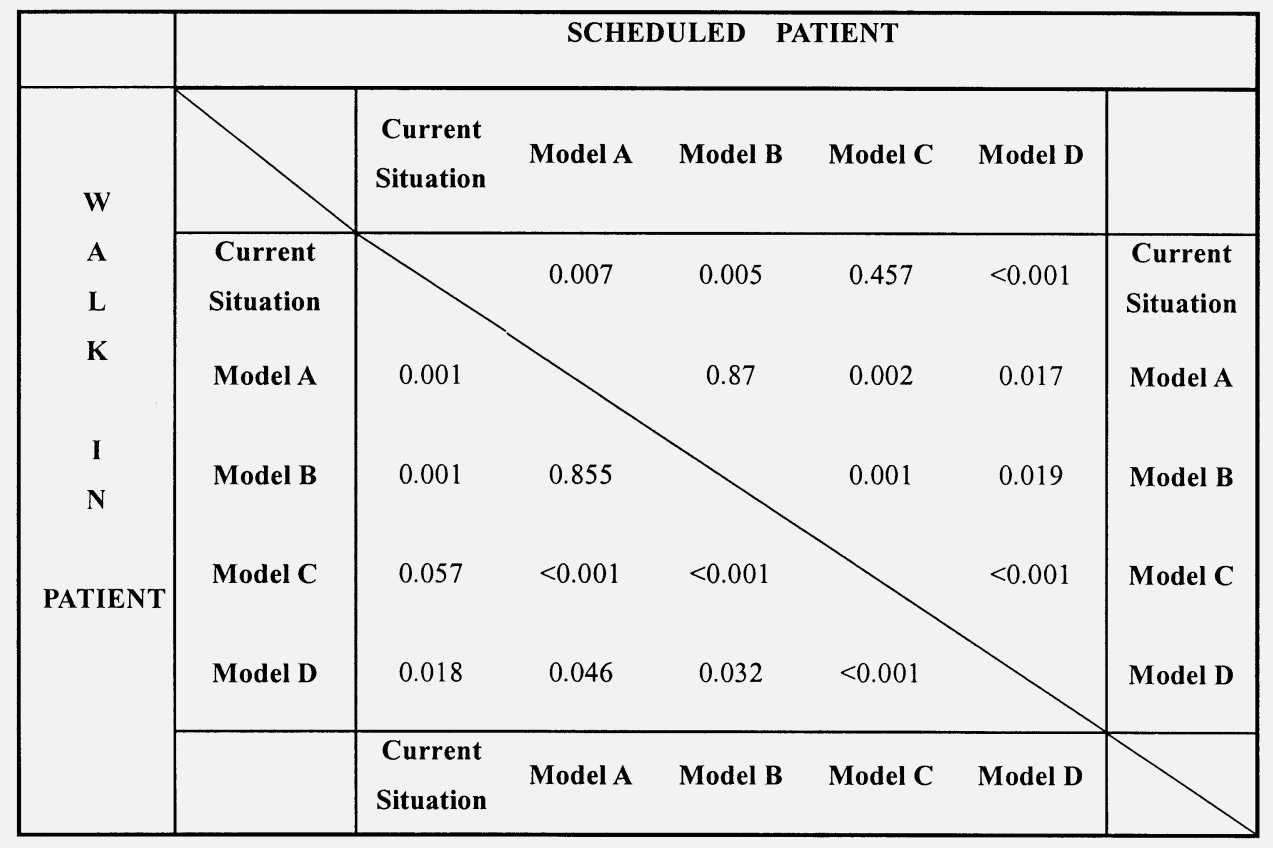

* Significance is defined as $p<0.01$

recommended interval times, we ran weeklong simulations which averaged 23.5 min (S.D.: 35.1 $\min$ ) of throughput time and $7.4 \mathrm{~min}$ (S.D.: 18.7 min) of waiting time. The scheduled patient spent less time in this simulated scenario in both throughput time and waiting time than under the current policy or alternative model A (see Tables 3 and 4). However, there were no significant differences between alternative model $D$ and other alternative models. All of the above had statistical significance $(P<0.01$; see Tables 5 and 6$)$.

\section{Conclusion and discussion}

One purpose of the preregistration strategy is to spread outpatient arrival times to produce a more even workload in clinics. Researchers observed that the patients did not come based on the appointment schedule in the study clinics or know when it was their turn to see a doctor. The present operating philosophy is "first in, first out": if the scheduled patient does not appear on time for his/ her turn, the next available patient receives consultation immediately. Under this system, patients believe that they can be seen earlier if they come earlier. Thus, patients do not have incentives to preregister for a clinic visit. This research utilizes the advantages of appointment in clinic operations. A more efficient scheduling policy is of great interest. When a clinic implements a stricter scheduling system, the goal is to lower the total throughput time, especially for scheduled patients. Based on this objective, the question is the following: "for scheduled patient arrival, what is the best time interval for effectively spreading outpatients but not resulting in longer working hours for physicians?" This research found that the appropriate appointment time interval for outpatient's arrival does not decrease patient-physician contact time, but it does permit walk-in patients to be scheduled as well. Moreover, the throughput and the waiting times are shortened for scheduled patients $(24.2$ and $8.0 \mathrm{~min}$, respectively). For walk-in patients, the throughput and waiting times are slightly longer than the current situation (46.2 min vs $39.4 \mathrm{~min}$ and $25.8 \mathrm{~min}$ vs 19.5 min, respectively; see Tables 3 and 4).

This research explored and predicted the behavior of the outpatient clinic after the implementation of new scheduling policies that require all scheduled patients to be present on time. The researchers found no significant difference in a patient's throughput time or waiting time despite assigning previous numbers to scheduled patients (alternative model B) or under alternative model A 
Table 6 Comparison matrix of waiting time using Student's $t$-test ( $P$-value) for current situation and models A-D

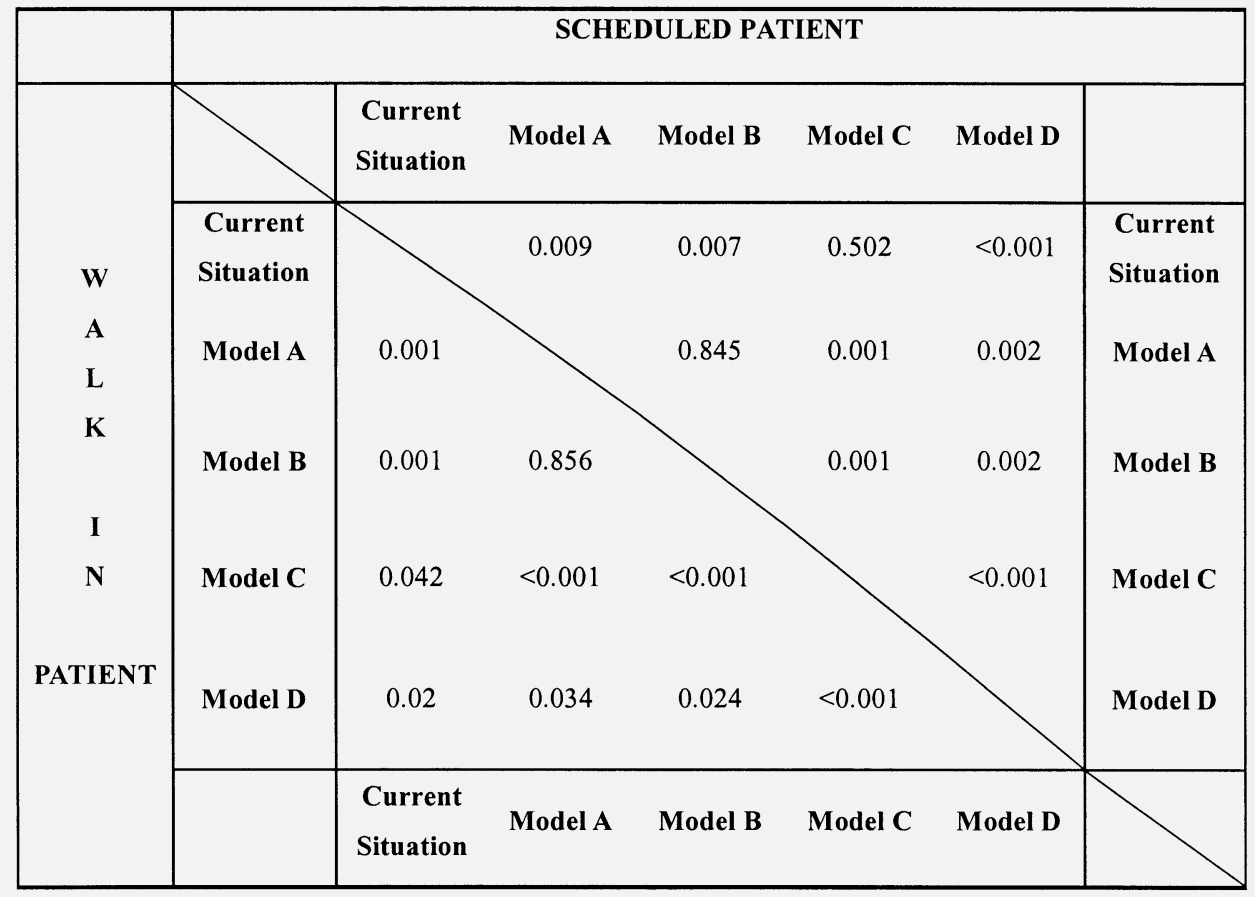

* Significance is defined as $p<0.01$

of the clinics. The alternative sequence for scheduled and walk-in patients (alternative model C: even numbers for scheduled and odd numbers for walk-ins) is expected to improve throughput and waiting times for walk-in patients and just slightly lengthen the time $(2 \mathrm{~min})$ for scheduled patients. This conclusion is based on the fact that in the subject clinic, walk-ins make up a greater proportion of all visits, and most of the walk-ins happen in the first 1 and $1.5 \mathrm{~h}$ of the day. Inevitably, patient's behavior might change by altering the policy. In future, the ongoing database such as the proportion of scheduled patients vs walk-in patients as well as the earliness and lateness of scheduled or walk-in patients can be used to simulate the clinic operation in order to provide for further modification of the scheduling system.

We have developed a simulation model that reflects the complexities of the health service processes, including the allowance for non-standard distributions for patient arrival times and individual station service times, the assignment of each patient to an individual physician, the ability to vary the patient mix (scheduled or walk-in), and the availability of multiple outcome possibilities. In our report, we demonstrate the model and its ability to estimate outcomes only under patient- oriented variables. It was limited not to explore the outcome resulted from changing staff numbers, adding resources, changing floor plan/arrangement of rooms (basement/first floor).

The duration for the time-motion study is aimed at collecting service time at various stations. It would be more defendable if we could collect data for a much longer time period, but with the impose of limited number (around 25 patient visits per clinic per section) of registrations in subject clinic, seasonal and holiday variation and capacity-saturated impact on waiting time may interfere just little on our measurements.

Evaluating the above-mentioned alternative models based on the performance indicators of throughput time and waiting time, we conclude the following:

1) For scheduled patients, model of various scheduled intervals (alternative model D) is the best. The first 20 numbers reserved for scheduled patients followed by even numbers only (alternative model A), and privileged numbers for scheduled ones (alternative model B) are about the same but better than scheduled/walk-in in alternative sequence (alternative model $\mathrm{C}$ ). 
Table 7 Recommended appointment time intervals in simulation model D

\begin{tabular}{llll}
\hline Clinic times & Situation $1(\mathrm{~min})$ & Situation $2(\mathrm{~min})$ & Recommended time interval (min) \\
\hline Monday (A.M.) & $7,9,11$ & $3,5,7$ & 7 \\
Monday (P.M.) & 3,5 & 5,7 & 5 \\
Tuesday (A.M.) & 3,5 & 5,7 & 5 \\
Tuesday (P.M.) & $7,9,11$ & 9,11 & 9 \\
Wednesday (A.M.) & 9,11 & $7,9,11$ & 9 \\
Wednesday (P.M.) & 3,5 & 5 & 5 \\
Thursday (A.M.) & 7,9 & 9,11 & 9 \\
Thursday (P.M.) & $5,7,9,11$ & $5,7,9,11$ & 5 \\
Friday (A.M.) & $5,7,9,11$ & $5,7,9,11$ & 5 \\
Friday (P.м.) & $5,7,9,11$ & $5,7,9,11$ & 5 \\
Saturday (A.M.) & $5,7,9,11$ & $5,7,9,11$ & 5 \\
\hline
\end{tabular}

Situation 1: the throughput time is less than the current situation; situation 2: the ratio of physicians' actual working hours to clinics' office hours is between 70 and $90 \%$; model D: scheduled patients assigned in successive by various time intervals.

2) For walk-in patients, scheduled/walk-in in alternative sequence (alternative model C) performs best.

3) Alternative model $D$ of various scheduled intervals results in $10.9 \mathrm{~min}(24.2 \mathrm{~min}$ vs 35.1 $\mathrm{min}$ ) less total throughput time and $10.8 \mathrm{~min}$ less waiting time $(8.0 \mathrm{~min}$ vs $18.8 \mathrm{~min})$ for scheduled patients. However, alternative model $D$ also results in increased total throughput time and waiting time by $6.9 \mathrm{~min}(46.2 \mathrm{~min}$ vs $39.4 \mathrm{~min}$ ) and $6.4 \mathrm{~min}$ ( $25.8 \mathrm{~min}$ vs 19.5 min), respectively, for walk-in patients in subject clinics.

4) Although both the total throughput time and waiting time slightly increase, alternative model $\mathrm{C}$ of scheduled/walk-ins in alternative sequence results in $2.1 \mathrm{~min}$ ( $37.2 \mathrm{~min}$ vs 35.1 min) more and $1.7 \mathrm{~min}$ ( $20.5 \mathrm{~min}$ vs $18.8 \mathrm{~min}$ ) more for scheduled patients, but $4.5 \mathrm{~min}$ (34.9 $\mathrm{min}$ vs $39.4 \mathrm{~min}$ ) less and $4.7 \mathrm{~min}(14.7 \mathrm{~min}$ vs $19.5 \mathrm{~min}$ ) less, respectively, for walk-in patients.

5) Overall, alternative model C of scheduled/ walk-ins in alternative sequence is highly suggested and alternative model $D$ of various scheduled intervals is second. Other models in our research are not desirable.

However, sometimes the long appointment time interval lengthens the physicians' total working hours and thus increases physician utilization, although it may not be cost effective. Resolving this problem by choosing an optimal appointment time interval depends on the philosophy of the healthcare provider.

A review of applicable previously published papers showed that three of them relate to this research, but they alter the appointment interval under the scheduled situation, which is quite different from the study system with high percentage of walk-in patients [9-11,15-17]. Because of different scenario-high percentage of walk-in patients - findings from those studies cannot apply to our system. This research provides the possible resolution to the unique and popular circumstances of the Asian healthcare system with mixed-registration-type. Mixed scheduling models for outpatient services should shed some light on managing such a system.

In the simulation world as it applies to industrial applications or to the healthcare environment, the difference is often in the degree of complexity. Unlike the industrial setting, the healthcare environment exhibits complex processing logic involving a wide variety of patients, products, and treatment regimens. As such, almost every processing problem, regardless of purpose, becomes an exercise in trying to limit the complexity of the problem rather than solving it. Accordingly, many modelers are forced to artificially alter or limit their design models to accommodate existing software or hardware constrains. The result is that models are often only gross approximations of the systems under study and produce outcomes with questionable validity. The advanced computer simulation software that we applied in this study constructs the elements to meet the needs of the complex programming requirements in the healthcare system, such as the simultaneous use of resources, multistage operational preemption, complex shift scheduling, and so on. As a result, many constraints a modeler of healthcare systems faces are eliminated. This positive development opens a whole 
new world of analytical possibilities for the healthcare industry.

\section{Acknowledgements}

The authors received financial support from the National Science Council (NSC 88-2416-H-002-022), Taiwan.

\section{References}

[1] G.M. Wang, The new trend in outpatient services in Taiwan, Hosp. J. 24 (3) (1991) 189-195.

[2] F. Huarng, M.H. Lee, Using simulation in outpatient queues: a case study, Int. J. Health Care Qual. Assur. No. 6 (1996) $21-25$.

[3] A.B. Bindman, G. Kevin, K. Dennis, Consequences of queuing for care at a public hospital emergency department, J. Am. Med. Assoc. 266 (8) (1991) 1091-1096.

[4] M. Babes, G.V. Sarma, Outpatient queues at the Ibn-Rochd Health Centre, J. Oper. Res. Soc. 42 (1991) 845-855.

[5] D.J. Worthington, Queuing models for hospital waiting lists, J. Oper. Res. Soc. 38 (5) (1987) 413-422.

[6] B.S. Bloom, A.M. Fendrick, Waiting for care-queuing and resource allocation, Med. Care 25 (2) (1987) 131-139.

[7] J.C. Benneyan, An introduction to using computer simulation in healthcare: patient wait case study, J. Soc. Health Syst. 5 (3) (1997) 1-15.

[8] A.G. Kalton, M.R. Singh, D.A. August, C.M. Parin, E.J. Othman, Using simulation to improve the operational efficiency of a multidisciplinary clinic, J. Soc. Health Syst. 5 (3) (1997) 43-62.

[9] J.E. Clague, P.G. Reed, J. Barlow, R. Rada, M. Clarke, R.H. Edwards, Improving outpatient clinic efficiency using computer simulation, Int. J. Health Care Qual. Assur. Inc. Leadersh Health Serv. 10 (4-51) (1997) 197-201.
[10] F. Hashimoto, S. Bell, Improving outpatient clinic staffing and scheduling with computer simulation, J. Gem. Intern. Med. 11 (3) (1996) 182-184.

[11] S.B. Boxerman, Simulation modeling: a powerful tool for process improvement, Best Pract. Benchmarking Healthc. 1 (3) (1996) 109- 117.

[12] L. Aharonson-Daniel, R.J. Paul, A.J. Hedley, Management of queues in out-patient departments: the use of computer simulation, J. Manage. Med. 10 (6) (1996) 50-58.

[13] R.H. Edwards, J.E. Clague, J. Barlow, M. Clarke, P.G. Reed, R. Rada, Operations research survey and computer simulation of waiting times into medical outpatient clinic structures, Health Care Anal. 2 (2) (1994) 164-169.

[14] J.S. Rakich, P.J. Kuzdrall, K.A. Klafehn, A.G. Krigline, Simulation in the hospital setting: implications for managerial decision making and management development, J. Manage. Dev. 10 (4) (1991) 31 - 37.

[15] J.L. Levy, B.A. Watford, V.T. Owen, Simulation analysis of an outpatient services facility, J. Soc. Health Syst. 1 (2) (1989) $35-49$.

[16] G. Romanin-Jacur, P. Facchin, Optimal planning of a pediatric semi-intensive care unit via simulation, Eur. J. Oper. Res. 2 (1987) 192-198.

[17] G. Vassilacopoulos, A simulation model for bed allocation to hospital inpatient departments, Simulation 45 (5) (1985) $233-241$.

[18] A.J. Allessandra, T.E. Grazman, R. Parameswaran, U. Yavas, Using simulation in hospital planning, Simulation 12, 30:2 (1978) 62-67.

[19] N.K. Kwak, P.J. Kuzdrall, H.H. Schmitz, The GPSS simulation of scheduling policies for surgical patients, Manage. Sci. 22 (1976) 982-989.

[20] A. Raout, M. Ben-Daya, Outpatient clinic's staffing: a case study, Int. J. Health Care Qual. Assur. Inc. Leadersh Health Serv. 10 (6-7) (1997) 229-232.

[21] MedModel software, Version 3.5, Promodel Corporation, Orem, UT.

[22] BestFit software, Version 2.0, Palisade Corporation, Newfield, NY.

Available online at www.sciencedirect.com

science 0 DiRect. 